\title{
¿NACER EN EL CAMPO - MORIR EN LA CIUDAD? EXCLUSIÓN Y EXPULSIÓN DE LOS JÓVENES DE ÁREAS RURALES DE AMÉRICA LATINA.
}

Resumen. En el presente artículo se presentan distintas fuentes de vulnerabilidad social de los jóvenes rurales en Latinoamérica. Su inserción tanto laboral como social y su paso a la "vida de adulto" se ve limitada por diferentes barreras (económicas, culturales, sociales). A su vez, las influencias de la globalización (tecnologías de la información y comunicación) llegan a todos los rincones del planeta y abre nuevas expectativas entre los jóvenes, las cuales parecen ser sólo alcanzables en las ciudades. De esta forma se pueden definir factores de "expulsión", que llevan a los jóvenes a tener que dejar su lugar de origen, y factores de "atracción", que les hacen codiciar una vida diferente - a veces sólo una ilusión - en las ciudades. Se presentan algunos aspectos de las dificultades de acceso a la tierra y de la renovación generacional en Latinoamérica. Finalmente se dan algunas recomendaciones aplicables a las políticas públicas a emprender.

Palabras claves: juventud rural, políticas de juventud, desarrollo rural, renovación generacional, vulnerabilidad social.

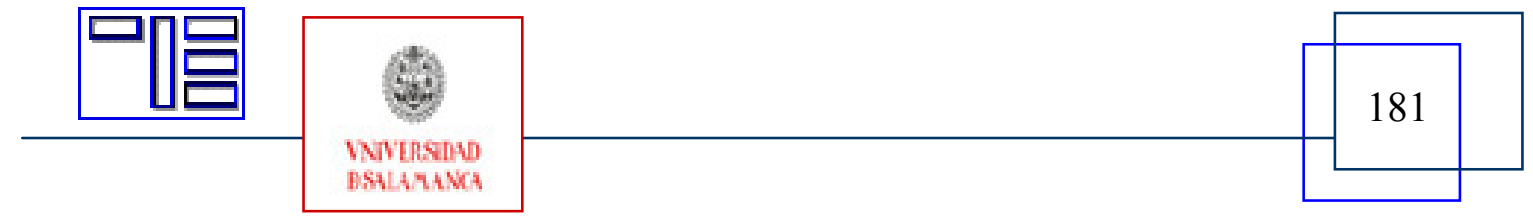




\section{BORN IN THE COUNTRYSIDE - DIE IN THE CITY? EXCLUSION AND EXPULSION OF YOUNG PEOPLE FROM LATIN AMERICAN RURAL AREAS.}

Abstract. This paper presents different sources of social vulnerability of the rural youths in Latin America. Different economic, cultural and social barriers limit the access of young people into the social and working context, as well as their first step into the "adulthood". Moreover, the influence of globalization (information and communication technologies), reaching every part of the world, opens up in the youths new expectations, which seem to be achievable only in the cities. In this frame two kinds of factors can be defined: "push-factors", driving youths out of their native areas, and "pull-factors", making them long for a different life (sometimes only illusory) in the city. Some aspects of the obstacles to land property access and to generational renovation in Latin America are here presented. Finally, some suggestions applying for the future public policies are given.

Key Words: rural youth, youth politics, rural development, generational renovation, social vulnerability.

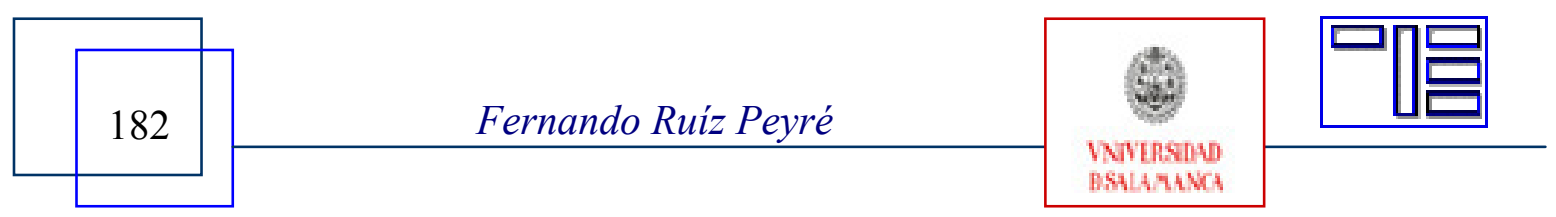




\section{NAÎTRE À LA CAMPAGNE - MOURIR EN VILLE?: EXCLUSION ET EXPULSION DE PERSONNES JEUNES DANS LES RÉGIONS RURALES DE L'AMÉRIQUE LATINE.}

Sommaire. Cet article présente différentes sources de vulnérabilité sociale des jeunes ruraux en Amérique Latine. Différentes barrières économiques, culturelles et sociales limitent l'intégration des jeunes dans le contexte social et de travail - ainsi qu'aux premiers pas vers une vie adulte.

De plus, l'influence de la mondialisation (technologies de l'information et de la communication) atteignent chaque coin de la planète et ouvrent de nouveaux espoirs aux jeunes, ce qui semble être uniquement possible dans les ville. De ce fait, peut être défini le facteur "expulsion" : poussant les jeunes à quitter leurs lieux d'origine et le facteur "attraction" : permettant une meilleure condition de vie - parfois illusoire - dans les villes.

Sont présentés ici quelques aspects de difficultés d'accès aux terres et de la rénovation générationnelle en Amérique Latine. Enfin, quelques recommandations sont données pour engager les politiques publiques.

Mots-clés: Jeunesse rurale, politique de jeunesse, développement rural, rénovation de génération, vulnérabilité sociale.

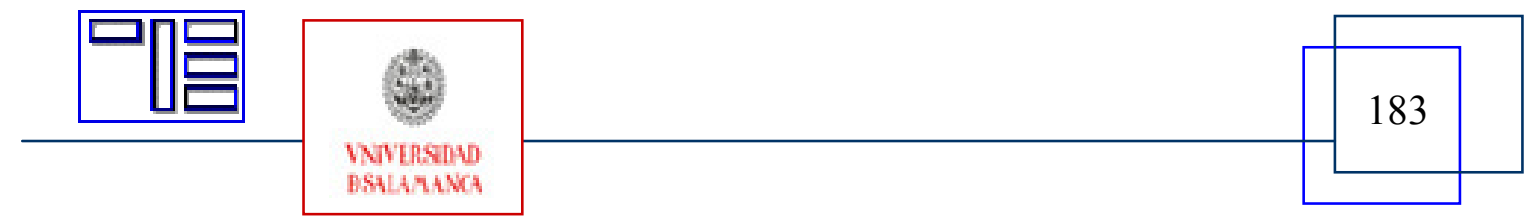


Fernando Ruiz Peyré

fernando.ruiz-peyre@student.uibk.ac.at

Universidad de Innsbruck (Austria)

\section{1.- INTRODUCCIÓN.}

La situación de los jóvenes toma cada vez más importancia en las discusiones sobre los llamados países en desarrollo. Esto se hace evidente por las cada vez más numerosas publicaciones sobre el tema, como por ejemplo el nuevo World Development Report 2007, dedicado exclusivamente a las "Nuevas Generaciones" y su importancia para el “desarrollo" (BANCO MUNDIAL, 2007).

Por otro lado, los cambios sociales producto de la globalización, así como nuevas tendencias en el campo latinoamericano, la llamada "nueva ruralidad", le dan a este grupo social una nueva significación y relevancia.

En el presente artículo se pretende analizar la situación de vulnerabilidad de los jóvenes en áreas rurales latinoamericanas, en especial el problema del éxodo rural y del acceso a la tierra.

Se presentarán primero aspectos conceptuales referentes a la juventud como objeto de estudio así como la diferenciación entre diferentes tipos de juventud. Se profundizará en las problemáticas del acceso a la propiedad de la tierra y en las prácticas de herencia (renovación generacional), aspectos claves en la decisión de permanencia o emigración por parte de los jóvenes. Finalmente, se presentará una serie de recomendaciones, útiles tanto para estudios similares en el tema así como para la definición de políticas públicas.

\section{2.- LOS JÓVENES COMO OBJETO DE ESTUDIO.}

Hasta hacer relativamente pocos años, la juventud prácticamente no era tenida en cuenta en los debates sobre los problemas de "desarrollo". La percepción de grupos con especiales vulnerabilidades se había limitado principalmente a la situación de los niños, de la mujer y de ciertas minorías culturales (por ejemplo de los pueblos indígenas) pero raramente se percibió a los jóvenes explícitamente en este sentido (ver Mayer, 2002, p. 19). En los últimos años, y debido a crecientes crisis y conflictos, el tema va ganando en resonancia en el marco de la cooperación internacional y bilateral para el desarrollo

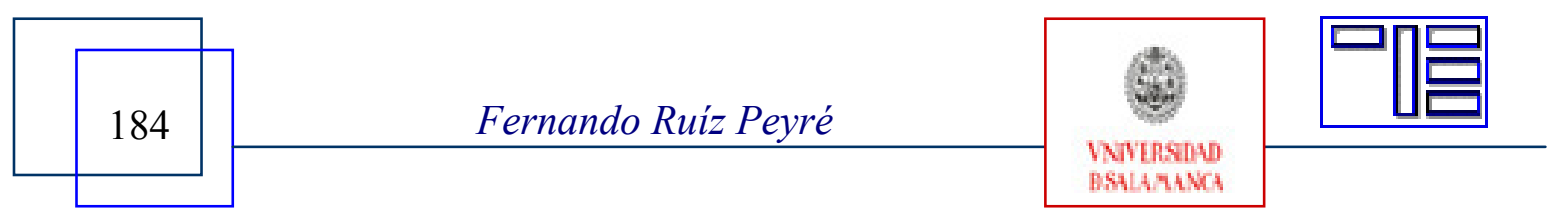


(Loewen, 2005, p. 12), así como para la planificación de políticas sociales en los países en desarrollo (Cepal, 2000).

Además de esta cierta falta de interés observada hasta el momento, la juventud tiende a ser vista como un "grupo problema" (Roche \& Tucker, 1997). Los jóvenes, sobre todo en los países en desarrollo, tienen una imagen negativa, relacionada por ejemplo con la violencia (pandillas callejeras), drogas, falta de expectativas, etc. Esta 'problematización' de la juventud, lleva a que los servicios sociales y pedagógicos se hayan orientado en lo que Roth llamó "servicio de reparación" de fallas ya presentes y no al cambio de las condiciones sociales que generan dichas fallas (Roth, 1983, p. 9).

Pero en los últimos años se ha abierto una nueva "dimensión" en el debate que pone a los jóvenes en el centro de las políticas de desarrollo (al menos en un plano discursivo). Numerosos autores e instituciones ven en las nuevas condiciones de globalización tecnológica y económica un notorio impulso al aporte potencial de los jóvenes al desarrollo de sus sociedades (CEPAL, 2000; Aparicio 2007). También es cierto que el nivel de formación promedio de los jóvenes de hoy supera en mucho el alcanzado por sus padres y tiene una tendencia creciente.

Pero si se analiza más en detalle se puede reconocer distintas "juventudes", las cuales se reparten en forma desigual las nuevas amenazas y oportunidades. De este modo, la situación de los jóvenes es una prueba más del predominio de un desarrollo fragmentado (Scholz, 2002; 2004), tanto en lo espacial como en lo social.

En una rápida conclusión podría decirse que los jóvenes de hoy, en el marco de una vertiginosa globalización, se encuentran en la paradoja de ser las víctimas de exclusión $\mathrm{y}$ falta de posibilidades por un lado y de ser los portadores de esperanza de cambio por el otro.

\subsection{El concepto de juventud.}

Existen numerosas definiciones de juventud que varían de acuerdo a la disciplina de la que provenga, del ámbito en el que se defina y de los objetivos que se persigan con ella. Pero la gran mayoría se asemejan al definirla como una etapa de la vida que comienza con la pubertad (aspecto biológico) y que termina con la asunción plena de las responsabilidades y la autoridad de un adulto (aspecto sociocultural) (ver Durston, 1998; Dirven, 2002; Romero Cabrera, 2004; entre otros).

Por motivos prácticos suele tomarse los 15 años como edad de inicio, debido a la información estadística (demográfica) disponible en la mayoría de los países. En otros casos (como para el Banco Mundial), se toma los 12 años como comienzo.

Para la edad tope existen mayores diferencias, ya que por tratarse en general de un límite sociocultural, es difícil definirlo a priori. Pero a fines prácticos la mayoría de los estudios fijan una edad que varía entre los 24 y los 29 años en la mayoría de los casos y

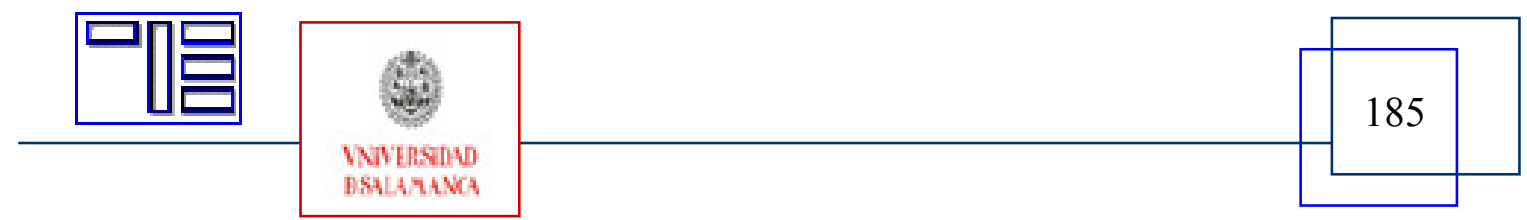


que en ciertos contextos (principalmente en Europa) se extiende hasta los 34 años de edad.

\subsection{Distintas juventudes.}

Rodriguez Vignoli (2001, p. 15 y ss.) reconoce cuatro grupos de jóvenes con características específicas: a) los estudiantes universitarios; b) la juventud popular urbana; c) las mujeres jóvenes; y d) los jóvenes rurales.

- Los estudiantes universitarios fueron los primeros jóvenes en ser considerados como grupo ya que siempre cumplieron con las condiciones para ser reconocidos como tales (Rodriguez Vignoli, 2001, p. 15). Se caracterizan por ser políticamente activos - sobre todo a partir de las revueltas estudiantiles de los años sesenta - y por estar relativamente bien organizados. El acceder a un nivel educativo elevado los coloca en una situación de privilegio que lo diferencia fuertemente de los otros grupos de jóvenes. Sin duda es este grupo uno de los "ganadores" de la globalización, es decir uno de los que más se beneficia de las ventajas generadas por los avances en las tecnologías de la comunicación e información.

- En oposición al anterior, se define la juventud popular urbana, llamada a veces la otra juventud (Rodriguez Vignoli, 2001, p. 16). Su irrupción social puede situarse en la década del setenta y se caracteriza por su falta de acceso a la educación superior y una situación general de exclusión. Se origina en los sectores más humildes de las grandes ciudades - muy extendidos en las urbes latinoamericanas - y se organizan en "pandillas" en los que sus miembros generan un proceso de identificación y pertenencia, en oposición a una sociedad que los aísla y excluye. También es la "juventud problema" que más preocupa a los especialistas, sobre todo porque es en este grupo donde se detectan las mayores tendencias a la violencia juvenil y al consumo de drogas. En combinación con otros tipos de "marginación" (por ejemplo de grupos de inmigrantes o minorías raciales) puede generar un cóctel explosivo como el observado en los suburbios de las ciudades francesas en el año 2005.

- El caso de las mujeres jóvenes presenta características muy marcadas, afectadas por una doble exclusión social, etaria y de género - y que puede llegar a ser triple en el caso de determinados sectores sociales -, sin identidad propia en los movimientos juveniles y tampoco en los de mujeres (Rodriguez Vignoli, 2001, p. 16). Este grupo se ve enfrentado con tradiciones enraizadas de una sociedad predominantemente machista - aunque con grandes diferencias de acuerdo al contexto cultural - y con una tendencia a ir ganando espacios de reconocimiento social. Uno de los sectores en los que ha realizado importantes avances, sobre todo en el contexto latinoamericano, es en el acceso a la educación media y

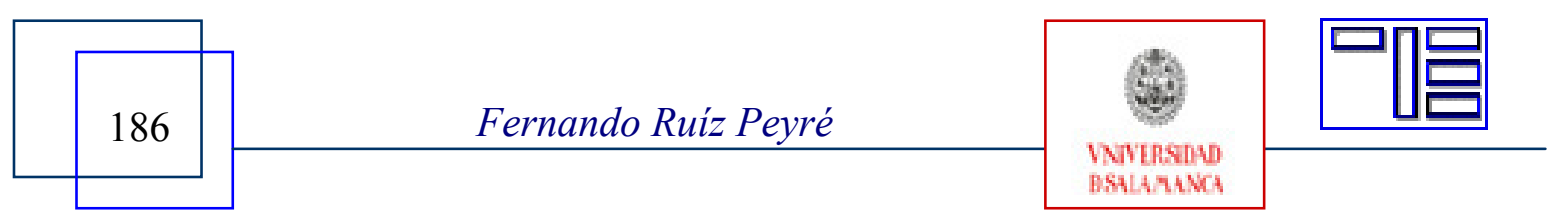


superior, en la que - en la mayoría de los países - ya no presenta importantes diferencias (WORLD BANK, 2007, p. 272).

- Este trabajo se ocupará principalmente de un cuarto grupo, de los jóvenes rurales, quizás una de las menos estudiadas de las 'juventudes', sobre todo teniendo en cuenta que el concepto de joven tal cual se entiende actualmente se originó en un contexto urbano. Este grupo de jóvenes quizás sea también el que más se encuentra expuesto a los cambios que trae consigo la globalización de las tecnologías de la información y comunicación, estando también cada vez más influenciados por la cultura urbana moderna (Rodríguez Vignoli, 2001, p. 16). Las condiciones especiales y las vulnerabilidades de este grupo serán analizadas en más detalle en el próximo apartado.

\subsection{Juventud rural como agente del desarrollo.}

Como ya se dijo, los y las jóvenes de áreas rurales presentan características particulares. De ellas se desprenden importantes potencialidades, las cuales son vistas con buenos ojos por quienes ocupan puestos de toma de decisión. Una de las mayores virtudes es el ya mencionado mayor nivel de educación que los jóvenes rurales alcanzan en la actualidad en comparación con sus padres. Como se observa en todos los países presentados en el gráfico 1, los hijos duplican el promedio de años de estudio de sus padres. También debe recalcarse que todos los países presentan por lo menos un promedio de 4 años de educación. Esto también se refleja a su vez en las tasas de analfabetismo, las cuales han descendido notablemente de una generación a otra (CEPAL, 2000). Este mayor nivel de educación trae consigo la ventaja de una mayor facilidad a la incorporación de nuevas tecnologías, las cuales pueden llevar a mejorar la productividad de los campos y también a una mejora en la calidad de vida.

Sin embargo debe quedar claro que "más educación" no lo es todo. Es importante tener en cuenta el contenido y la calidad de la educación formal. "En muchos aspectos, prevalece una visión demasiado urbana de la educación, que no siempre apunta a las necesidades del mundo rural" (Castillo, 2001, p. 128). A su vez, debe tenerse presente la educación "informal", ya que es por este canal por el que se transmiten todavía una gran parte de los conocimientos relacionados con la actividad agrícola.

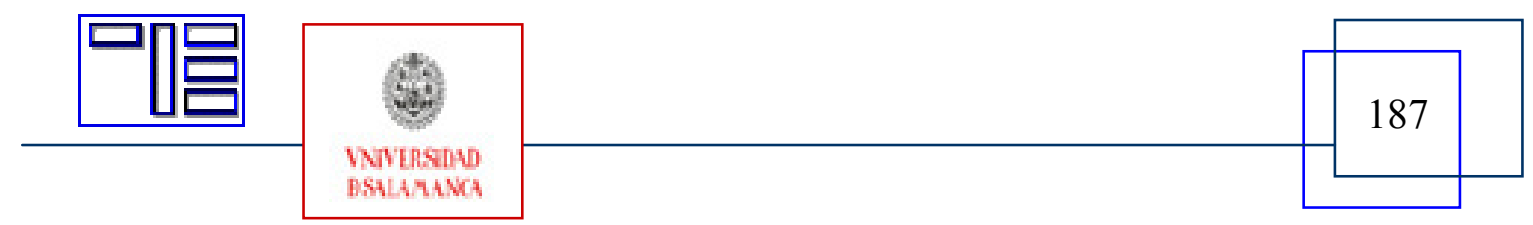


Revista Electrónica Teoría de la Educación.

Educación y Cultura en la Sociedad de la Información.

http://www.usal.es/teoriaeducacion

Vol. 9. No 2. Mayo 2008

Gráfico 1. Promedio de años de estudio de lo jóvenes de 20 a 24 años de edad y de sus padres para las áreas rurales de países de América Latina (selección de países).

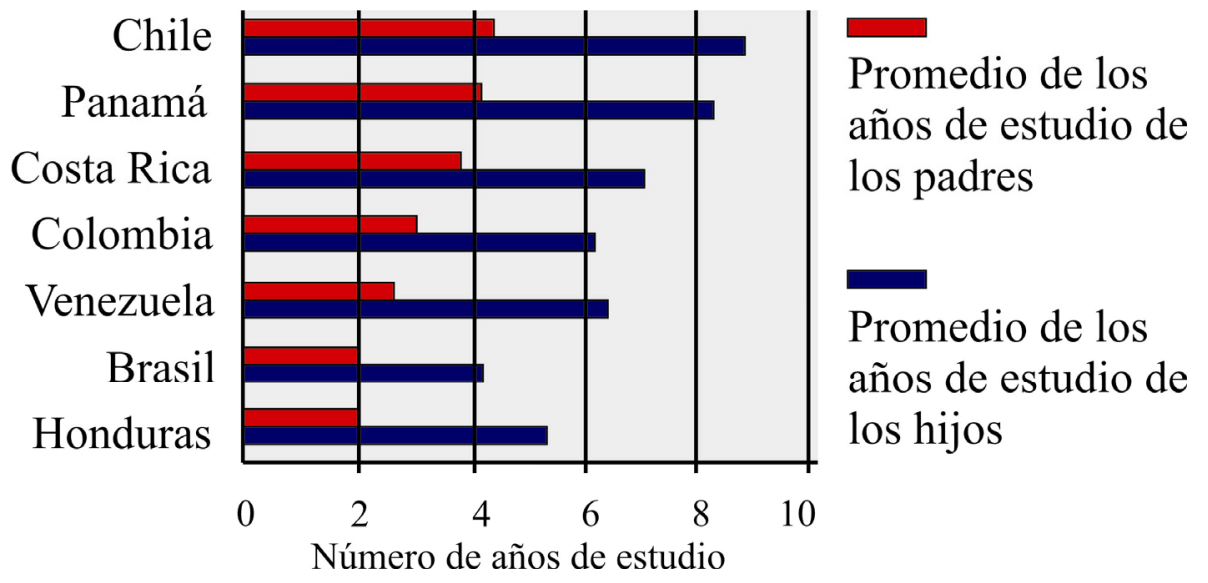

Fuente: Durston, 2001, p.104; en base a datos de la CEPAL.

Por otro lado, no puede olvidarse el déficit educativo en comparación con los jóvenes urbanos, los cuales alcanzan aún niveles muy superiores, tanto en cantidad de años de formación como en la calidad de la misma (CEPAL, 2000).

Además de la educación, otra dimensión clave para los jóvenes es, por supuesto, el aspecto laboral, que suele ser el determinante en la posibilidad de permanencia o no en lugar de origen (Duhart, 2004). Más allá del trabajo en el predio familiar, los jóvenes rurales comienzan generalmente una actividad remunerada mucho antes que sus pares urbanos (CEPAL, 2000).

Una opción, la cual en muchas ocasiones está ligada a la migración, son los trabajos temporales o de estación en regiones más o menos alejadas. Este tipo de trabajo tiene la ventaja de un ingreso inmediato y de aportar nuevas experiencias. Sin embargo suele ser "un trabajo mal pagado y duro, que genera inseguridad y dependencia, no siendo una alternativa de proyecto de vida que fortalezca las capacidades y autoestima de los jóvenes rurales" (Duhart, 2004, p. 128).

Otra alternativa que toma cada vez más importancia es la de los empleos rurales no agrícolas, los cuales crecieron a tasas del 3,5\% a principio de los noventas, mientras que los empleos en actividades agrícolas presentaron una disminución de hasta el 4,5\% anual en igual período (Schejtman, 1999, p. 25). Este desarrollo presenta nuevas oportunidades, las cuales pueden significar una opción para la inclusión laboral de población joven, quizás aprovechando sus mayores niveles educacionales ya mencionados.

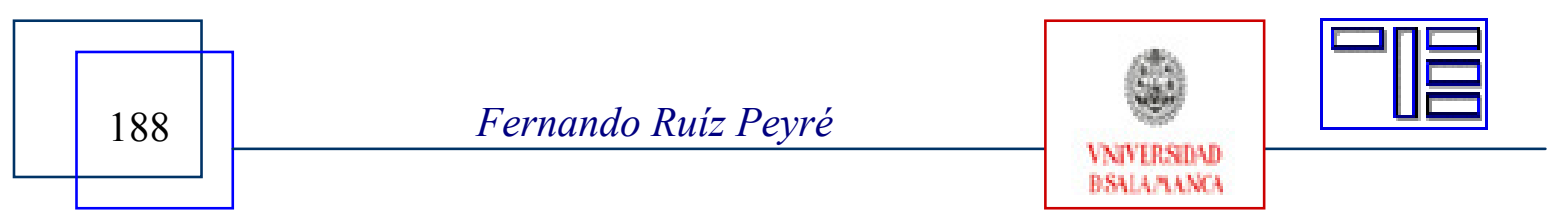




\section{3.- EL ÉXODO RURAL.}

Los movimientos migratorios del campo a la ciudad han sido estudiados por diferentes disciplinas (geografía, sociología, economía, etc.) con diferentes enfoques por largo tiempo.

Prácticamente todos los espacios rurales latinoamericanos sufrieron una etapa de fuerte éxodo de población como consecuencia, principalmente, de cambios en los modos de producción agrícola, que generalmente fue acompañada por un aumento de mano de obra industrial en los centros urbanos. Si bien esta dinámica ha cambiado y las relaciones campo-ciudad han sido resignificadas por las innovaciones tecnológicas, puede afirmarse que el éxodo rural continúa activo (Duhart, 2004; Dirven, 2000).

Y son los precisamente jóvenes los que generalmente dejan el campo y para migrar hacia las ciudades. Pero esto no se debe ya a la mencionada demanda de mano de obra urbana, sino más bien a la falta de posibilidades de permanencia para gran parte de las nuevas generaciones (Dirven, 2000, 16). También se puede explicar por las barreras existentes que "impiden a los jóvenes tanto su inserción activa en [el] sistema [como] la asunción plena de los roles de adultos” (Castillo, 2000, p. 6).

Esto es lo que Patricio Castillo en su trabajo sobre las juventudes rurales en Chile llama "Sistema de Flujo Generacional Cerrado", en el que destaca barreras intergeneracionales de distinta magnitud (Gráfico 2). Esas barreras tienen diferentes connotaciones (económicas, sociales, culturales), las cuales se presentan con mayor fuerza en el paso de la juventud a la edad adulta. Algunas de las formas que suelen tomar son dificultades para el acceso a la tierra, a créditos o incluso al asesoramiento técnico. Como resultado, una gran parte de los jóvenes se ve prácticamente obligada a dejar el campo y buscar oportunidades zonas urbanas. Mientras más fuerte es la barrera, mayor es la tasa de emigración.

\section{Gráfico 2. Sistema de flujo generacional.}

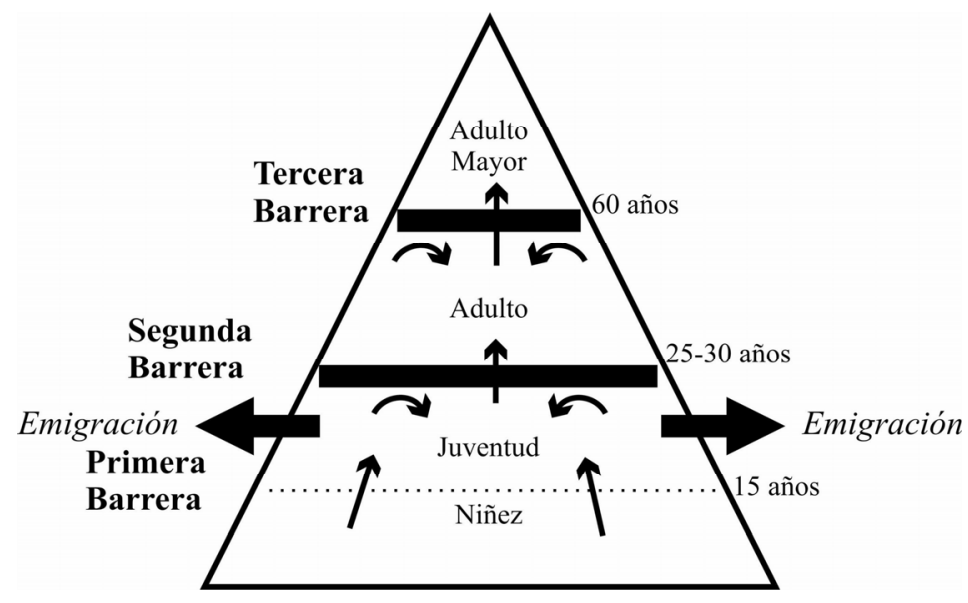

Fuente: Castillo, 2000, p.6.

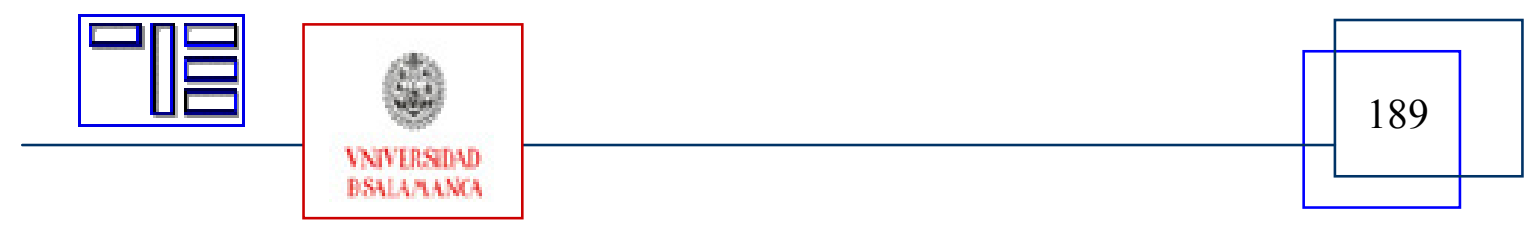


Como resultado de esta combinación de limitaciones, se calcula que aproximadamente un tercio de los jóvenes dejan el campo para dirigirse a las ciudades (DIRVEN, 2000, p. 16). De este modo la inversión realizada, tanto por el estado como por las familias, en educación y formación no puede ser puesta en práctica en el ámbito rural y es transferida a los espacios urbanos.

Lo que debe quedar claro es que no se trata de evitar la emigración en sí misma, que de hecho puede también ser vista como apertura de oportunidades o de mejora social. Pero ésta comienza a ser un problema serio "cuando asoma como un peligro real el abandono de fincas económicamente viables, o cuando comunidades enteras puedan desaparecer del mapa, por falta de [...] jóvenes suficientes para reproducir los hogares" (Durston, 2001, p.114).

\subsection{El acceso a la tierra}

Una de las mayores barreras que se detectan en el paso de la edad joven a la adulta en el contexto rural es el acceso a la tierra. Esta dificultad queda clara si se observa la distribución de las propiedades de acuerdo a la edad del productor en Chile (Gráfico 3), situación que se observa en la mayoría de los países de la región (Castillo, 2001).

Como resultado a esta difícil inserción laboral agrícola se detectan diferentes alternativas, las cuales dependen tanto de factores socio-culturales-económicos como personales y regionales. También se observan claras diferencias en la solución de este conflicto de acuerdo al género. Una gran parte de las mujeres jóvenes encuentra trabajo en las ciudades cercanas, generalmente en empleos de bajo nivel, principalmente en el sector de servicios. Los varones jóvenes pueden buscar empleo como peones rurales hasta poder asumir la dirección de un predio.

\section{Gráfico 3. Distribución de las propiedades de acuerdo a la edad del productor.}

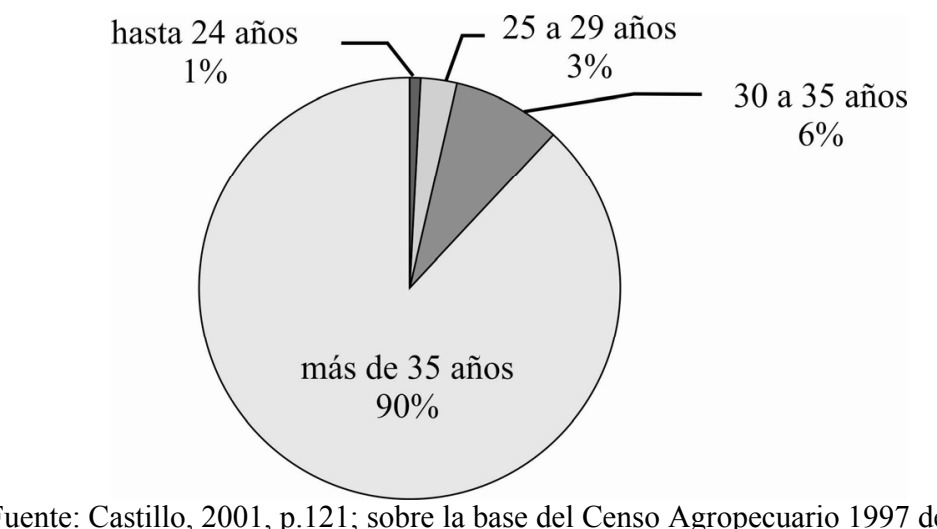

Fuente: Castillo, 2001, p.121; sobre la base del Censo Agropecuario 1997 de Chile.

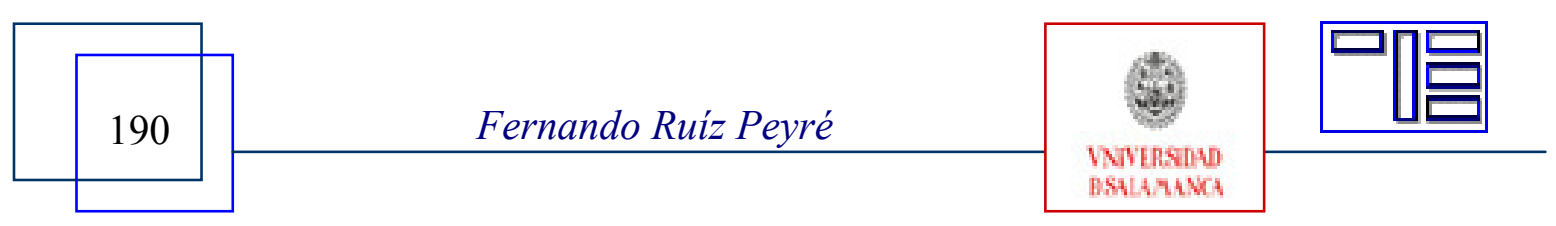


Lo que cabe preguntarse es entonces, hasta qué punto sirve la educación recibida, en la que se promueve incluir las nuevas tecnologías, nuevas formas de administración o el manejo de nuevos cultivos, si esos conocimientos podrán ser puestos en práctica recién después de 15 ó 20 años (Castillo, 2001). Por supuesto, no se debe olvidar el valor de la experiencia adquirida con la edad, ni de poner en duda su importancia. Se trata principalmente de analizar críticamente las inversiones hechas por el estado y de pensar las posibilidades de inserción de las nuevas generaciones. Es decir: de planificar el futuro.

\subsection{La renovación generacional}

La herencia de la tierra (y el traspaso en general de la propiedad de una generación a la siguiente) juega un papel fundamental en acceso a la tierra para las nuevas generaciones. Como es sabido, la agricultura familiar - predominante en gran parte de los espacios rurales latinoamericanos - presenta características particulares que la diferencia de otras formas productivas. En general se basa en la fuerza de trabajo familiar, la cual continúa de generación en generación. Por otro lado, "sus dimensiones no permiten que de ella dependa más de una familia sin pauperizarla" (Dirven, 2002, p. 23). La subdivisión de las propiedades de generación en generación, observada en diferentes lugares de Latinoamérica, da como resultado el llamado "minifundio" con consecuencias sociales y económicas devastadoras y de difícil recuperación.

Dirven analiza en su obra "Las prácticas de herencia de tierras agrícolas: ¿una razón más para el éxodo de la juventud?" la situación actual en Latinoamérica en el traspaso de la propiedad de una generación a la otra (Gráfico 4). En ella se observa una herencia demorada de las propiedades y tiene como resultado una concentración de las mayores explotaciones en manos de personas mayores de 60 años de edad, justamente cuando las "fuerzas" productivas comienzan a descender. El traspaso sucede entonces de forma abrupta, generalmente después de la muerte del jefe del predio. Esta situación predominante se basa en costumbres de herencia que dependen de las culturas y tradiciones locales. Sin embargo, se observan en este sentido también nuevas tendencias y cambios producidos por las influencias de la "globalización" y el avance del modo de vida urbano en las zonas rurales.

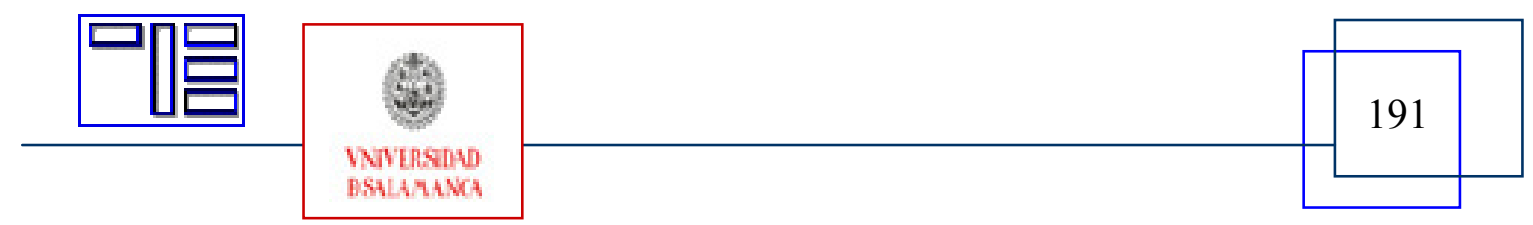




\section{Gráfico 4. Traspaso de las propiedades de una generación a la otra. Situación actual (izquierda) y situación deseable (derecha).}
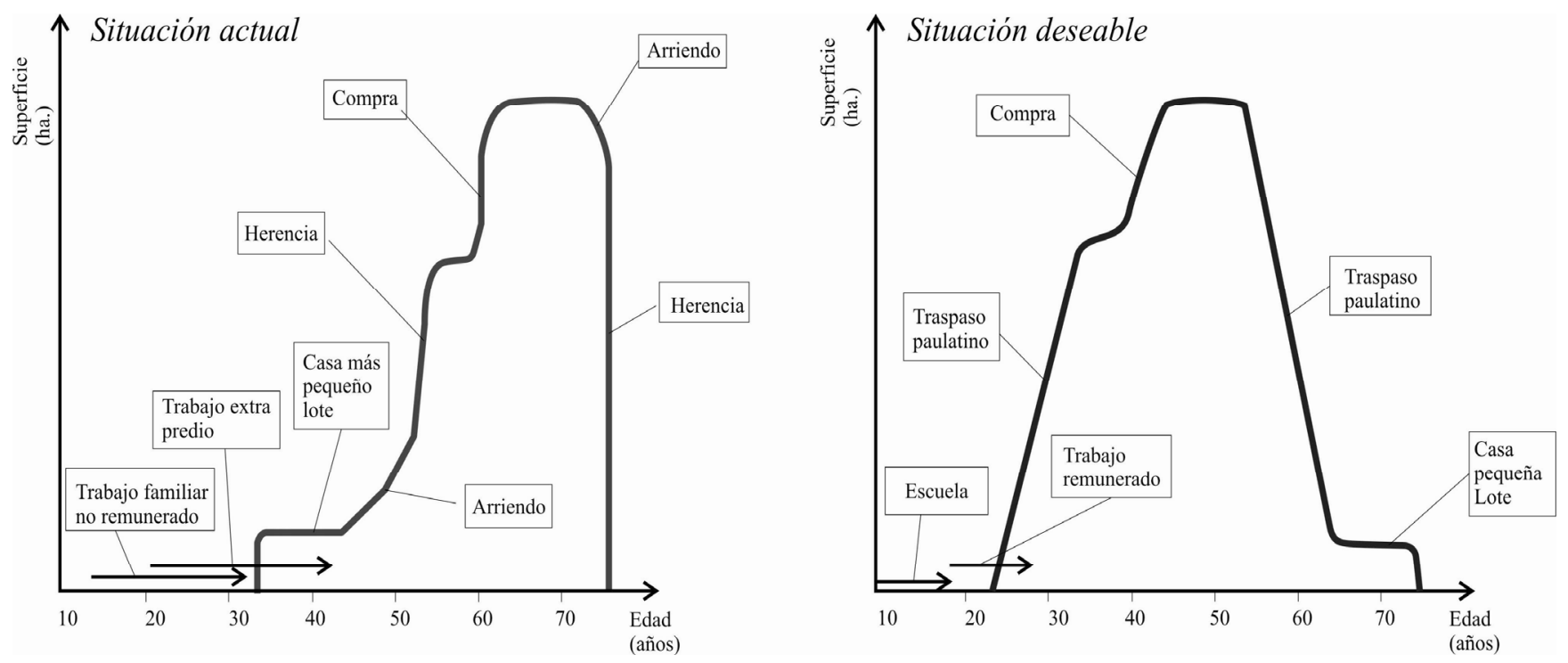

Fuente: Dirven, 2002, p. 24.

La situación deseable presentada por Dirven se vería con un traspaso paulatino más temprano de las propiedades a la generación siguiente, de tal modo que permita que los jóvenes asuman la dirección de las propiedades en sus años más productivos. En esta etapa sería importante el acceso al crédito y al asesoramiento técnico que acompañen el comienzo de la explotación agrícola. A su vez, propone un "retiro" más suave y la aseguración de una pequeña renta que acompañe a las personas mayores.

Pero un cambio de este tipo no puede pensarse sin una adaptación de los sistemas de jubilaciones y pensiones, los cuales presenten fuertes déficit - si no son inexistentes -en la mayoría de los espacios rurales latinoamericanos.

Está claro que la dinámica presentada presenta una fuerte carga cultural y que su cambio sólo es posible en un plazo relativamente largo. Sin embargo, ese no es motivo para no promover políticas públicas tendientes a revertir situaciones indeseables.

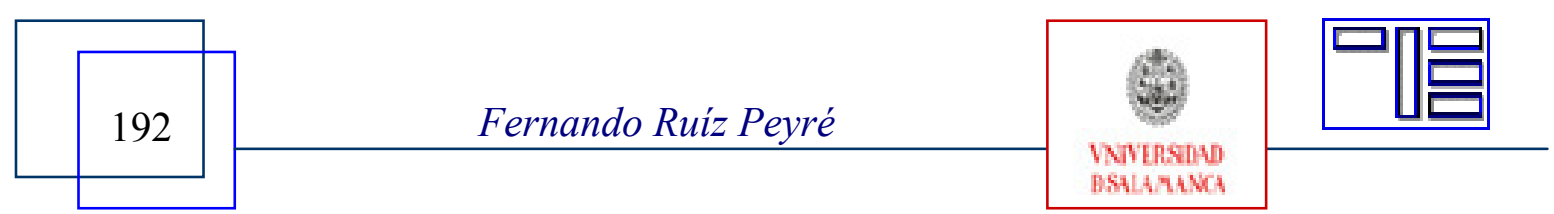




\section{4.- CONCLUSIÓN Y ALGUNAS RECOMENDACIONES.}

Recapitulando lo presentado en este artículo queda claro que la población joven rural presenta importantes potencialidades, pero que para que puedan ponerse en marcha deben superarse primero numerosas limitaciones.

También ha quedado claro lo importante de tener en cuenta el enfoque etario para las políticas de desarrollo, sobre todo en un espacio rural latinoamericano que se caracteriza por una población cada vez más envejecida. Crear una nueva línea de créditos debe adaptarse, por ejemplo, a las demandas reales de los más jóvenes. Y la edad de acceso a nuevos programas de jubilaciones y pensiones toma un significado clave: si es muy alta agudizará el problema, si es más baja ayudará a una renovación generacional más acelerada.

¡No se trata sólo de los jóvenes tengan que quedarse obligadamente en el campo! Por su puesto que deben tener la posibilidad de elegir: "la alternativa de la residencia urbana es un derecho de toda persona" (Durston, 2001, pp. 114). Pero el objetivo debe ser que tengan un abanico real de opciones entre las que puedan elegir. Es decir, entre otras cosas, que el joven debe alcanzar una educación y formación que le permitan un desarrollo profesional/personal tanto en el espacio rural como en el urbano. Para alcanzar esto - que no se logra actualmente - está claro que debe haber políticas específicas, que tengan en cuenta los numerosos y variados factores que influyen en ese proceso de decisión (algunos de los cuales fueron presentados en este breve artículo).

Y por último, pero no por eso menos importante, debe promoverse una participación activa de los jóvenes en la definición de las políticas que les atañen. Sin poner exageradas ambiciones en ella, la participación puede concebirse como un instrumento más en el camino que los propios jóvenes deben recorrer para llegar a la vida adulta.

En los tiempos actuales se viven cambios sociales vertiginosos. El mundo moderno influye cada vez más en las estructuras sociales tradicionales, las cuales reaccionan de diferentes maneras. También la relación campo-ciudad toma nuevos significados. "Las fronteras entre lo "rural" y lo "urbano" se tornan cada vez más imprecisas en lo concerniente a las diferentes idealizaciones y proyectos de los jóvenes" (Carneiro, 1998, pp.115). Estas nuevas relaciones deben ser promovidas y mejoradas de tal modo que ambos lados se beneficien.

\section{5.- BIBLIOGRAFÍA.}

APARICIO, P. C. (2007). Los jóvenes y la vertiginosa transformación educativa, social y laboral. Una aproximación socio educativa a la realidad de la/os jóvenes argentina/os, Studi sulla Formazione, IX, 2006, Univeristá di Firenze, pp. 86-106.

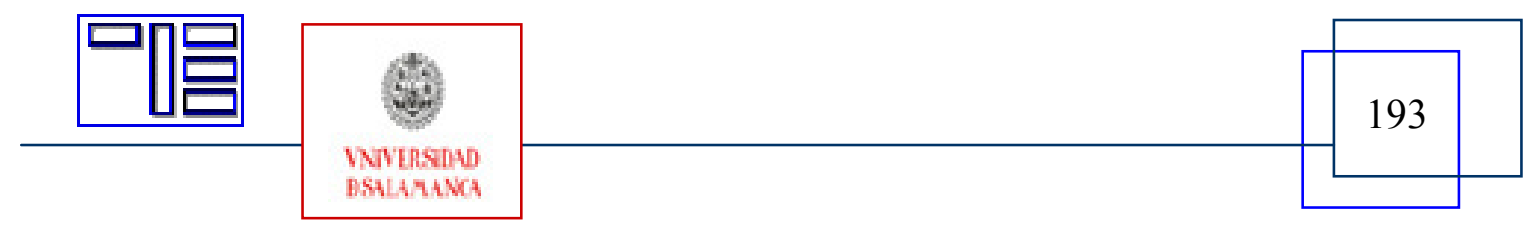


BOHLE, H.-G. (2001). Vulnerability and Criticality: Perspectives for Social Geography. Update IHDP, 2/01, pp. 1-5.

CARNEIRO, M. J. (1998). O ideal rurbano: campo e cidade no imaginário rurais, en TEIXERA DA SILVA, F.C. et al. (eds.) Mundo Rural e Politica, Rio de Janeiro: Ed. Campus, pp. 95-118.

CASTILlO, P. (2000). Juventudes rurales como agentes de desarrollo del sector. Ventajas y barreras para la acción, Santiago de Chilo, Tesis de grado (sin publicar), Facultad de Agronomía, Universidad de Chile.

CASTILlO, P. (2001). La juventud rural del Cono Sur: agentes de cambio y desarrollo para la superación de la pobreza rural, en CEPAL Protagonismo juvenil en proyectos locales: lecciones del cono sur, Santiago de Chile: CEPAL, pp. 111131.

CEPAL (2000). Juventud, población y desarrollo: problemas, posibilidades y desafíos, Serie Población y Desarrollo, 6, Santiago de Chile: CEPAL.

DIRVEN, M. (2000). Strategien zur Eindämung der Landflucht in Lateinamerika, Entwicklung + ländlicher Raum, 5/2000, pp.16-19.

DIRVEN, M. (2002). Las prácticas de herencia de tierras agrícolas: ¿una razón más para el éxodo de la juventud?, Serie Desarrollo Productivo, 135, Santiago de Chile: CEPAL.

DURSTON, J. (1998). Juventud y Desarrollo Rural: marco conceptual y contextual, Serie Políticas Sociales, 28, Santiago de Chile: CEPAL.

ESPINDOLA, D. (2005). Organizaciones y movimientos juveniles rurales en cinco países del Mercosur: (Argentina, Brasil, Chile, Paraguay y Uruguay). Situación actual $\mathrm{y}$ propuestas para su fortalecimiento, Revista Electrónica Latinoamericanade Estudios sobre Juventud, 1 (1).

LOEWN, B. (2005). Jugendlichen im Fokus von Bildung und Beschäftigung: Herausforderungen für die Entwicklungszusammenarbeit, en Loewen, B. y Overwien, B. (eds.) Jugendlichen stärken - Entwicklungspolitische Ansätze und Perspektiven für Bildung und Beschäftigung, Frankfurt a.M.: IKO-Verlag für Interkulturelle Kommunikation, pp. 9-20.

MARX, B. (1999). Soziale Entwicklung in ländlichen Regionen. Ein theoretischer und empirischer Bezugsrahmen für ein Konzept sozialer Regionalentwicklung für die Zielgruppen Frauen und Jugend, Münster/Freiburg: LIT/Lambertus-Verlag.

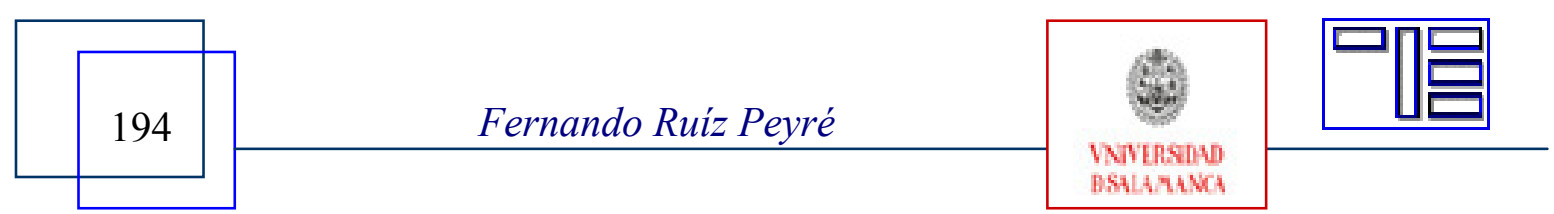


MAYER, M. (2002). Jugend, Verwundbarkeit und Soziale Diskriminierung Lebenschancen und Konfliktpotentiale Ländlicher Jugendlicher in Sri Lanka, Geographica Helvetica, 57 (1), pp. 19-33.

ROCHE, J. \& TUCKER, S. (1997). Youth in Society. Contemporary Theory, Policy and Practice, London: SAGE.

RODRIGUEZ VIGNOLI, J. (2001). Vulnerabilidad y grupos vulnerables: un marco de referencia conceptual mirando a los jóvenes, Serie Población y Desarrollo, 17, Santiago de Chile: CEPAL.

ROMERO CABRERA, J. I. (2004). La modernización agraria en el Uruguay: los jóvenes rurales, una asignatura pendiente, en GIARRACCA, N. \& LEVY, B. (eds.) Ruralidades Latinoamericanas. Identidades y Luchas Sociales, Buenos Aires: CLACSO, pp.163-201.

ROTH, L. (1983). Die Erfindung des Jugendlichen, Manchen: Juventa Verlag.

SCHEJTMAN, A. (1999). Las dimensiones urbanas en el desarrollo rural, Revista de la CEPAL, 67, pp.15-32.

SCHOLZ, F. (2002). Die Theorie der fragmentierenden Entwicklung, Geographische Zeitschrift, 88 (1), pp. 1-20.

SCHOLZ, F. (2004). Geographische Entwicklungsforschung, Stuttgart: Gebrüder Bontraeger Verlagbuchhandlung.

SEN, A. (2000). Development as Freedom, Nueva York: Knopf \& Borzoi Books.

WORLD BANK (2007). Development and the Next Generation. World Development Report 2007, Washington DC: World Bank.

Para citar este artículo puede utilizar la siguiente referencia:

RUIZ PEYRÉ, Fernando (2008). ¿Nacer en el campo morir en la ciudad? exclusión y expulsión de los jóvenes de áreas rurales de América Latina.En APARICIO, Pablo (Coord.) Desde la diversidad hacia la desigualdad: ¿destino inexorable de la globalización? [monográfico en línea]. Revista Electrónica Teoría de la Educación: Educación y Cultura en la Sociedad de la Información. Vol. 9, $\mathrm{n}^{\mathrm{o}}$ 2. Universidad de Salamanca. [Fecha de consulta: dd/mm/aaaa]. $<$ http://www.usal.es/ teoriaeducacion/rev_numero_09_02/n9_02_ruizpeyre.pdf $>$ ISSN 1138-9737

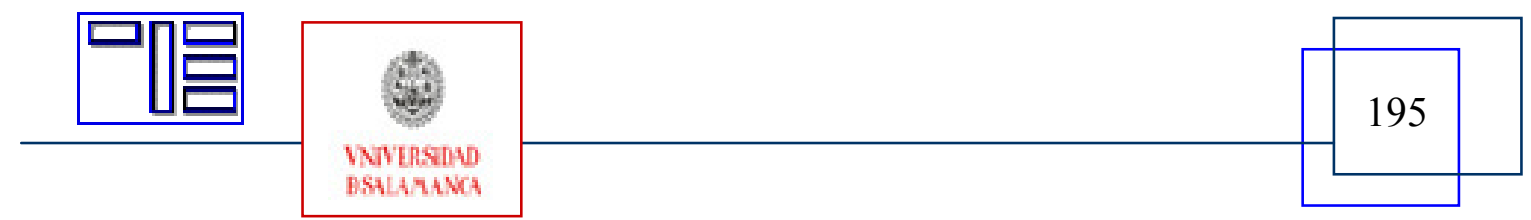

